Short Communication

\title{
Low-cost internal controls for detection of Giardia cysts in water samples
}

\author{
Melissa Solano Barquero*, Eric Morales Mora, Luz Chacón Jiménez, Erick Cordero Jara, \\ Liliana Reyes Lizano, Kenia Barrantes Jiménez, Rosario Achí
}

Sección Infección Nutrición, Instituto de Investigaciones en Salud (INISA), Universidad de Costa Rica, 11501-2060 San José, Costa Rica

\section{A R T I C L E I N F O}

\section{Keywords:}

Giardia

Internal control

Flocculation

Water

\begin{abstract}
A B S T R A C T
Giardia cysts stained with hot carbolfuchsin were used as internal controls in a concentration method for surface water samples. The morphological integrity of stained cysts and the stain's stability and intensity were tested with each of the chemical reagents used in the aluminum sulfate flocculation method. No alterations in morphology or color were noted. The stained cyst preparation has a low cost, high stability, and suitability for both light and immunofluorescent microscopy, making it affordable to researchers in low- and middle-income countries.
\end{abstract}

Giardiasis is a worldwide distributed parasitic infection included since 2004 in the "Neglected Diseases Initiative", a heterogeneous group of parasitic, bacterial and viral infections that occur mostly in developing countries, related to poverty, lack of access to public and medical services [1,2]. Efstratiou et al., [3] reported 142 waterborne diarrhea outbreaks caused by Giardia spp. from 2011 to 2016, from a limited number of developed countries. In middle and low income countries there are scarce reports of Giardia outbreaks due to the lack of governmental systems for documentation of water parasitic protozoa in water supply [3].

Giardia infection is acquired by the ingestion of cysts present in contaminated water or food $[1,4]$. Giardia cysts are highly resistant in the aquatic environments and to disinfectants, such as the usual chlorine concentration applied in water treatment plants [5]. Furthermore, Giardia cysts are highly infectious; as few as 10 cysts may cause infection [5]. Consequently, it is relevant to determine the presence of cysts in source waters intended for human consumption, where cyst detection and enumeration can inform on treatment barriers that need to be in place, so that the risk of infection stays below tolerable levels [6].

The United States Environmental Protection Agency (USEPA) Method 1623 [7] is the most validated and widely used for the detection of Giardia and Cryptosporidium in water samples [8,9]. However, alternative, less expensive methods, such as the formalin-ether concentration and flocculation have also been proposed [10].

Methods used for detection of Giardia cysts in water samples must be validated and the recovery efficiency determined at the performing laboratory [8,9]. To this end, several internal control standards are available commercially, including EasySeed ${ }^{\mathrm{TM}}$, Easy $\mathrm{PC}^{\mathrm{TM}}$, Waterborne
AccuSpike $^{\mathrm{TM}}[11,12]$, and ColorSeed ${ }^{\mathrm{TM}}$ [13]. Typically, these products contain Giardia and Cryptosporidium (oo)cysts that have been quantified and certified by the manufacturer. ColorSeed ${ }^{\mathrm{TM}}$, an internal control cysts stained with red fluorescence dye, allows differentiation between the cysts naturally present in the water samples (green fluorescence) from the internal control cysts (red) [8,9]. The main disadvantages of all these controls are their high cost and low stability (loss of fluorescence with time).

The purpose of this study was to evaluate carbolfuchsin stained Giardia cysts preparation, as an alternative low cost and stable internal control, to be used in concentration, recovery, identification and enumeration stages of Giardia cysts detection in water samples.

Fresh stool samples $(<12 \mathrm{~h})$ positive for Giardia duodenalis were obtained from the Clinical Laboratory of the Universidad de Costa Rica. The parasites were concentrated by the Ritchie (ether-formol) method [14]. and stored in $5 \%$ formalin at $4^{\circ} \mathrm{C}$ until used (Fig. 1A).

Prior to staining, the cysts were washed with distilled water three times, counted by Neubauer chamber, and distributed in aliquots containing 100,000 cysts, which were centrifuged ( $1774 \mathrm{G}, 6 \mathrm{~min}$, at room temperature) and the supernatants discarded. The pellets were stained with $1 \mathrm{~mL}$ of the following dyes: Wright, Giemsa, Lugol 1\%, methylene blue $0.3 \%$, green malachite $2 \%$, safranin $2 \%$ and crystal violet $2 \%$, for $10,30,120 \mathrm{~min}$ and overnight, respectively. None of the dyes were appropriate, because the cysts lost color after being inoculated in distilled water and incubated for $30 \mathrm{~min}$ at room temperature.

Giardia cysts were subsequently stained using $50 \mu \mathrm{L}$ of carbolfuchsin $3 \%$ and $2 \mathrm{~mL}$ of distilled water, in duplicate. The suspension 1 was heated on an open flame, steamed but not to the boiling point (to avoid precipitates), for $5 \mathrm{~min}$. The suspension 2 was incubated for $5 \mathrm{~min}$ at

\footnotetext{
* Corresponding author.

E-mail address: melissa.solano_b@ucr.ac.cr (M. Solano Barquero).
} 


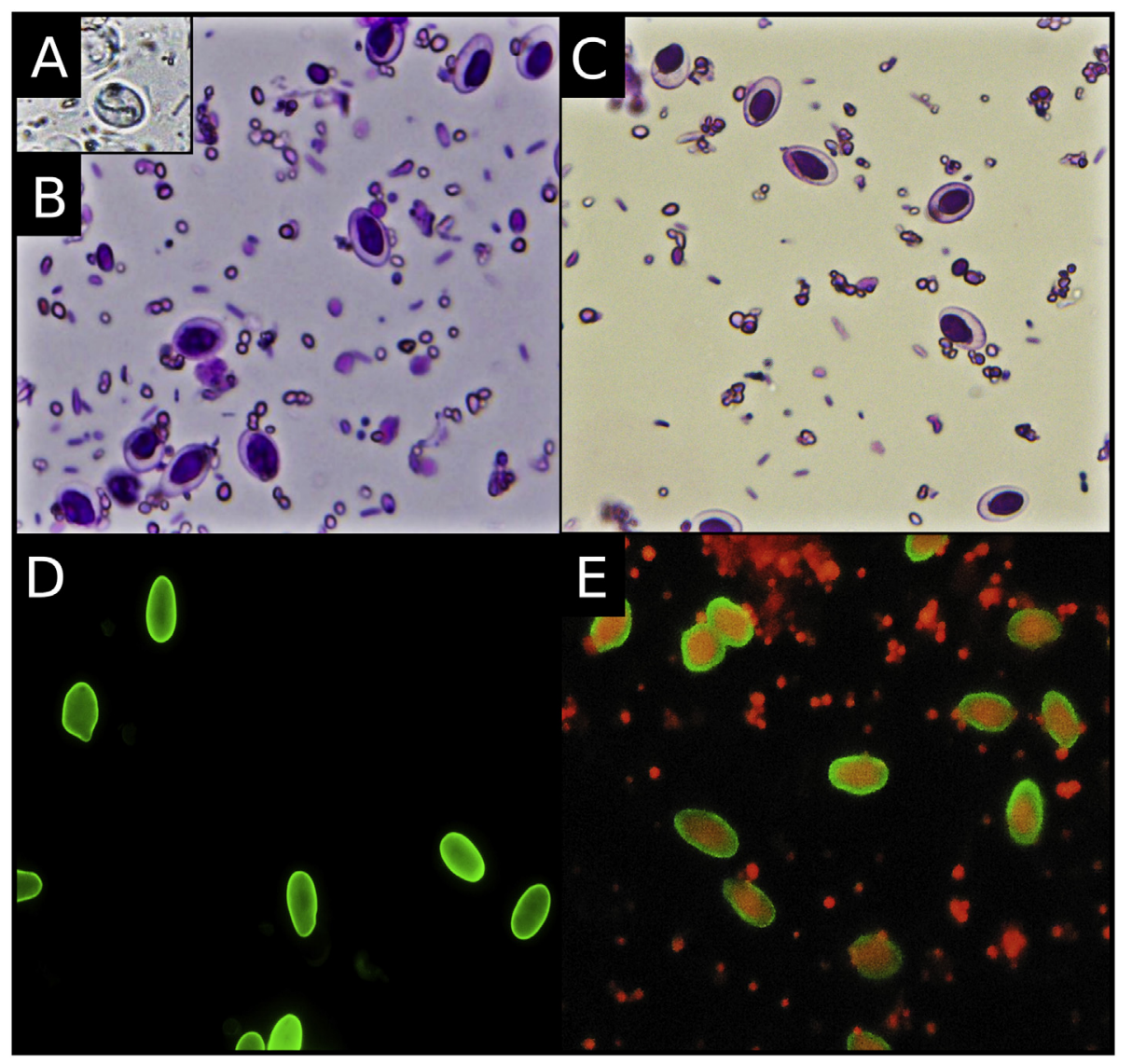

Fig. 1. Giardia cysts: A. Giardia cysts with saline solution under light microscopy, B. Giardia cysts stained under light microscopy, C. Giardia cysts stained with carbolfuchsin after one year of storage under light microscopy, D. Giardia cysts stained with fluorocrome (IFA-FICT) under fluorescence microscopy. E. Giardia cysts stained with carbolfuchsin and with fluorocrome (IFA-FICT) under fluorescence microscopy. Samples were observed over light microscope and fluorescence microscope $40 \mathrm{X}$ magnification.

room temperature. Both suspensions were then washed and centrifuged (1774 G, $6 \mathrm{~min}$, at room temperature) three times. Under light microscopy, the cysts from suspension 1 showed a uniform intense fuchsia color with a transparent halo, ovoid morphology, and internal structures were not observed (Fig. 1B). The cysts from suspension 2 did not show a uniform coloration, there were cysts with intense fuchsia color, and internal structures were not observed and other cysts were less stained with visible internal structures.

Cysts from the suspension 2 lost color after being inoculated in distilled water and incubated for $30 \mathrm{~min}$ at room temperature, so its use was discarded. Staining intensity did not decrease in cysts from suspension 1 after being kept over $48 \mathrm{~h}$ in distilled water at room temperature. Those carbolfuchsin stained Giardia cysts (SGC) stored at $4{ }^{\circ} \mathrm{C}$ in formalin $5 \%$ were stable for over 1 year without losing the retention capacity of the dye upon water contact (Fig. 1C). Carbolfuchsin is a dye that is routinely used heated for the staining of other microorganisms like Cryptosporidium sp. and Mycobacterium sp. in the Ziehl-Neelsen technique [15].

Using the software CellSens ${ }^{\circledR}$ of CLX Olympus ${ }^{\circledR}$ microscope model BX43F, the morphology and diameter of concentrated Giardia unstained cysts and SGC (after a year of storage at $4{ }^{\circ} \mathrm{C}$ in $5 \%$ formalin), were examined (Table 1). In order to simulate the conditions during the concentration process, 100 SGC were analyzed for morphology and size (longer diameter) after being incubated with each of the flocculation reagents individually for 20 days at $4{ }^{\circ} \mathrm{C}$ and following the complete flocculation method, as described by Karanis and Kimura [16]. (Table 1). To ensure the quality of the SGC staining, 5000 cysts were examined in six repetitions (30,000 cysts in total). All the cysts showed uniform coloration; only $0.1 \%$ (44 cysts) showed weak staining.

Flocculation is an inexpensive technique with several steps and reagents that could decrease cysts recovery rate [17]. Also, the agitation could disrupt cell membranes in some organisms. In other cases, ions as $\mathrm{Fe}+2$ and $\mathrm{Al}+3$ may distress cell membranes and cause lysis [18]
Table 1 shows the effect of each flocculation reagents and the complete flocculation process on SGC. The morphology and size of the Giardia cysts were similar in all cases, differences in size not being statistically significant by ANOVA test $(p<0.0001)$. SGC maintain their color with these adverse conditions and show more stability than the 4 months reported by commercial controls.

In addition, the SGC were tested by immunofluorescence assay (FITC-IFA). Immunofluorescence stained SGC showed apple-green walls with an red cytoplasm (Fig. 1E) while unstained control cysts showed brilliant apple-green fluorescence in both, walls and cytoplasm, as described by US-EPA [7] (Fig. 1D).

The variability of water conditions and sample characteristics make it essential to use carefully planned and executed positive and negative controls [19]. Also, Giardia concentrations in surface water are near the limit of detection and not uniformly distributed [20]. for this reason the use of internal control is essential.

The SGC were used as internal controls in a field trial of aluminum sulphate flocculation for the analysis of $10 \mathrm{~L}$ of river water and $10 \mathrm{~L}$ of drinking water [16] and concentrated using immunomagnetic separation (IMS) of sediments, with the Dynabeads ${ }^{\circledast}$ GC-Combo kit according to manufacturer's instructions.

Recovered cysts were visualized by both light microscopy and by direct immunofluorescence (IFA). Recovered SGC showed the expected morphology and fluorescence pattern by IFA, as well as the expected morphology and stain by light microscopy. As a consequence, SGC are adequate as internal control for field trials.

Standard methodologies for detection and enumeration of Giardia spp. are expensive, usually requiring filtration, immunomagnetic separation and immunofluorescence assay steps [21]. This translates into high costs for both equipment and reagents. The high cost of internal controls could hinder method optimization [19].

In this paper we provide an inexpensive (less than $\$ 1$ per test) and stable method for preparing Giardia internal controls, useful for the 
Table 1

Comparison of the effect of flocculation reagents on morphology, diameter and percentage of discoloration in SGC.

\begin{tabular}{|c|c|c|c|c|c|c|}
\hline \multirow[t]{2}{*}{ Cyst } & \multirow[t]{2}{*}{ Conditions } & \multicolumn{2}{|l|}{ Morphology } & \multirow[t]{2}{*}{ Mean size $(\mu \mathrm{m})$} & \multirow[t]{2}{*}{$\mathrm{CV}$} & \multirow[t]{2}{*}{ Weakly colored cysts (\%) } \\
\hline & & Regular membrane cysts counted (n) & Spike membrane cysts counted (n) & & & \\
\hline \multirow[t]{6}{*}{$\mathrm{SGC}^{\mathrm{a}}$} & Distilledwater & 90 & 10 & 12.9 & 6.9 & 12 \\
\hline & $P B S T$ & 90 & 10 & 13.0 & 8.4 & 0 \\
\hline & Aluminum sulfate & 86 & 14 & 12.6 & 11.8 & 7 \\
\hline & Citric acid & 88 & 12 & 12.4 & 11.1 & 0 \\
\hline & Mixture of flocculation reagents ${ }^{b}$ & 83 & 17 & 12.7 & 11.4 & 5 \\
\hline & Complete flocculation process ${ }^{c}$ & 99 & 1 & 11.6 & 8.7 & 0 \\
\hline \multirow[t]{2}{*}{ Control } & Concentratedunstainedcysts & 100 & 0 & 10.4 & 8.3 & - \\
\hline & SGC after 1 year of storage & 93 & 7 & 11.3 & 15.3 & 0 \\
\hline
\end{tabular}

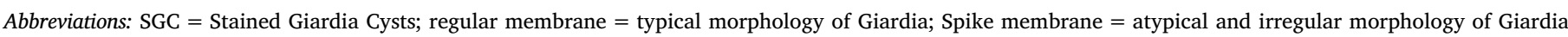
$\mathrm{CV}=$ coefficient of variation; PBST $=$ phosphate buffered saline with TWEEN $^{\circledR} 20$

a The SGC were analyzed after 20 days at $4{ }^{\circ} \mathrm{C}$ in the different reagents (distilled water, PBST, aluminum sulfate, citric acid, mix of flocculation reagents) and after all the flocculation process (1 day at room temperature).

b Mixture of flocculation reagents at the concentrations indicated by Karanis and Kimura, [16].

c All the flocculation process described by Karanis and Kimura, [16].

analysis of environmental water. Our control is versatile for its use in different methodologies as flocculation, immunomagnetic separation, light microscopy and immunofluorescence microscopy. The implementation of SGC can be a useful and inexpensive tool, particularly in low income countries, where efforts are needed to implement Giardia detection in water samples.

\section{Acknowledgements}

To the Scientific Publication Workshop of the Instituto de InvestigacionesenSalud (INISA) University of Costa Rica/University of Miami (UM), Florida and to Harlan Wolfe and Alberto Simhon for their recommendations in the preparation of this manuscript.

\section{Funding/support}

Vicerrectoría de Investigación, Universidad de Costa Rica.

\section{Declarations of interest}

None.

\section{References}

[1] L. Savioli, H. Smith, A. Thompson, Giardia and Cryptosporidium join the "neglected diseases initiative", Trends Parasitol. 22 (2006) 203-208, https://doi.org/10.1016/ j.pt.2006.02.015.

[2] F.M. Rosado-García, M. Guerrero-Flórez, G. Karanis, M.C. Hinojosa, P. Karanis, Water-borne protozoa parasites: the Latin American perspective, Int. J. Hyg. Environ. Health 20 (2017) 783-798, https://doi.org/10.1016/j.ijheh.2017.03.008.

[3] A. Efstratiou, J.E. Ongerth, P. Karanis, Waterborne transmission of protozoan parasites: review of worldwide outbreaks - an update 2011-2016, Water Res. (2017) 14-22, https://doi.org/10.1016/j.watres.2017.01.036.

[4] E.A. Adam, J.S. Yoder, L.H. Gould, M.C. Hlavsa, J.W. Gargano, Giardiasis outbreaks in the United States, 1971-2011, Epidemiol. Infect. 144 (13) (2016) 2790-2801, https://doi.org/10.1017/S0950268815003040.

[5] D. Carmena, Waterborne transmission of Cryptosporidium and Giardia: Detection, surveillance and implications for public health, in: A. Mendez-Vila (Ed.), Current Research, Technology and Education Topics in Applied Microbiology and Microbial Biotechnology, Formatex, Badajoz, 2010, pp. 3-141 http://www.formatex.info/ microbiology2/3-14.pdf.

[6] S. Regli, J. Rose, C. Haas, C. Gerba, Modeling the risk from Giardia and viruses in drinking water, Res. Technol. (1991) 76-84.

[7] United States Environment Protection Agency (EPA), Method 1623: Cryptosporidium and Giardia in Water by Filtration/IMS/FA, https://www.epa.gov/sites/ production/files/2015-07/documents/epa-1623.pdf, (2005), Accessed date: 14 March 2018.

[8] C.L. DiGiorgio, D.A. Gonzalez, C.C. Huitt, Cryptosporidium and Giardia recoveries in natural waters by using Environmental Protection Agency method 1623, Appl. Environ. Microbiol. 68 (2002) 5952-5955, https://doi.org/10.1128/AEM.68.12. 5952-5955.2002.

[9] M.I. Sato, A.T. Galvani, J.A. Padula, A.C. Nardocci, M. de Souza-Lauretto, M.T. Razzolini, E.M. Hachich, Assessing the infection risk of Giardia and Cryptosporidium in public drinking water delivered by surface water systems in Sao Paulo State, Brazil, Sci. Total Environ. 442 (2013) 389-396.1, https://doi.org/10. 1016/j.scitotenv.2012.09.077.

[10] F. Lora-Suarez, R. Rivera, J. Triviño-Valencia, J.E. Gomez-Marin, Detection of protozoa in water samples by formalin/ether concentration method, Water Res. 100 (2016) 377-381, https://doi.org/10.1016/j.watres.2016.05.038.

[11] R.M.B. Franco, R. Rocha-Eberhardt, R. CantusioNeto, Occurrence of Cryptosporidium oocysts and Giardia cysts in raw water from the Atibaia River, Campinas, Brazil, Re. Inst. Med. Trop. S. Paulo 43 (2001) 109-111, https://doi.org/ 10.1590/S0036-46652001000200011.

[12] K. Helmi, S. Skraber, L. Leblanc, L. Hoffmann, H.M. Cauchie, Comparison of three methods to concentrate Giardia cysts and Cryptosporidium oocysts from surface and drinking waters, Water Sci. Technol. 62 (2010) 196-201.

[13] T. Wohlsen, J. Bates, B. Gray, M. Katouli, Evaluation of five membrane filtration methods for recovery of Cryptosporidium and Giardia isolates from water samples, Appl. Environ. Microbiol. 70 (4) (2004) 2318-2322, https://doi.org/10.1128/AEM 70.4.2318-2322.200.

[14] L.S. Ritchie, An ether sedimentation technique four routine stool examinations, Bull. U. S. Army Med. Dept. 8 (1948) 326 http://stimson.contentdm.oclc.org/cdm/ ref/collection/p15290coll6/id/3672.

[15] World Health Organization (OMS), Manual of Basic Techniques for a Health Laboratory, Second Edition, WHO Library, Geneva, 2003.

[16] P. Karanis, A. Kimura, Evaluation of three flocculation methods for the purification of Cryptosporidium parvum oocysts from water samples, Appl. Environ. Microbiol. 34 (6) (2002) 444-449, https://doi.org/10.1046/j.1472-765X.2002.01121.x.

[17] M. Lepesteur, S. Blasdall, N.J. Ashbolt, Particle dispersion for further Cryptosporidium and Giardia detection by flow cytometry, Lett. Appl. Microbiol. 37 (2003) 218-229, https://doi.org/10.1046/j.1472-765X.2003.01381.x.

[18] J. Hou, Z. Yang, P. Wang, C. Wang, Y. Yang, X. Wang, Changes in Microcystis aeruginosa cell integrity and variation in microcystin-LR and proteins during Tanfloc flocculation and floc storage, Sci. Total Environ. 626 (2018) 264-273, https://doi. org/10.1016/j.scitotenv. 2018.01.074.

[19] A. Efstratiou, J. Ongerth, P. Karanis, Evolution of monitoring for Giardia and Cryptosporidium in water, Water Res. 123 (2017) 96-112, https://doi.org/10.1016/ j.watres.2017.06.042.

[20] J.E. Ongeth, ICR SS protozoan data site-by-site: a picture of Cryptosporidium and Giardain U.S. surface water, Environ. Sci. Technol. 47 (2013) 10145-10154, https://doi.org/10.1021/es4027503.

[21] A.A. Escobedo, P. Almirall, L.J. Robertson, R.M. Franco, K. Hanevik, K. Mørch, S. Cimerman, Giardiasis: The ever-present threat of a neglected disease, Infect. Disord. Drug Targets 10 (2010) 329-348, https://doi.org/10.2174/ 187152610793180821. 